\title{
Which prescriptive screening programmes are worth while?
}

\author{
JOCELYN M CHAMBERLAIN
}

From the South West Thames Regional Cancer Organisation, Block E, Royal Marsden Hospital, Sutton, Surrey SM2 5PT

SUMmaRY Prescriptive screening is defined as screening for diseases solely for the benefit of the individuals participating in the programme. Currently used screening programmes are examined at each stage of life - antenatal, postnatal, school age, adult life, and old age. It is concluded that most disorders for which screening can be confidently recommended for the benefit of the individual are those occurring in children: screening infants for phenylketonuria, hypothyroidism, visual and auditory impairment, and probably congenital dislocation of the hip. Subject to parents' informed consent to participate, antenatal screening for Down's syndrome and neural tube defects is effective in high risk populations. Screening programmes to detect in school children visual and auditory defects and dental diseases are worth while. Two major problems arise in most programmes-firstly, how to achieve a high compliance rate of the population in attending for $\stackrel{\infty}{\rightarrow}$ screening and accepting subsequent treatment; secondly, how to determine what treatment, if any, should be offered to borderline cases. Further evaluation of these problems is required for many programmes.

Screening people who are apparently well in order to detect unrecognised disease can be of value to the individuals so diagnosed if early treatment is able to improve their prognosis, and to society at large if their identification leads to primary prevention to protect other people from becoming affected. Screening solely for the benefit of individuals participating in the programme has been termed "prescriptive" screening, ${ }^{1}$ with the implication that this is something which the doctor prescribes for his patients because it may do them good. But, in considering which programmes should be implemented as health policy, it is not sufficient merely to know that screening does good, one must assess whether it does enough good to outweigh its disadvantages. ${ }^{2}$ This economic concept of comparing benefits and costs is not new; it was implicit in the first of the 10 precepts of screening listed by Wilson and Jugner $^{3}$ that the disease should be an important health problem. A few years later Cochrane and Holland were more explicit in their conclusion that, even if screening for porphyria was effective it was not worth while ${ }^{4}$ as the disease was so rare in the UK.

The severity and frequency of the disease, and the ability of early treatment to improve its prognosis, are not, however, the only aspects to be considered in a full evaluation. The table lists various benefits, setting against each its attendant disadvantages. The first and most important benefit is an improvement in prognosis for some, but not necessarily all, cases detected at screening. Identification of cases already too advanced to benefit from early treatment is certainly no advantage and may do a disservice to these patients by making them aware of their condition for longer, and possibly subjecting them to unpleasant but, in the event, ineffective treatment.

Benefits and disadvantages of screening

\begin{tabular}{ll}
\hline Benefits & Disadvantages \\
\hline $\begin{array}{l}\text { Improved prognosis for some cases } \\
\text { detected by screening }\end{array}$ & $\begin{array}{c}\text { Longer morbidity for cases whose } \\
\text { prognosis is unaltered }\end{array}$ \\
$\begin{array}{l}\text { Less radical treatment which cures } \\
\text { some early cases }\end{array}$ & $\begin{array}{c}\text { Overtreatment of questionable } \\
\text { abnormalities }\end{array}$ \\
$\begin{array}{l}\text { Resource savings } \\
\begin{array}{l}\text { Reassurance for those with negative } \\
\text { test results }\end{array}\end{array}$ & $\begin{array}{c}\text { False reassurance for those with } \\
\text { false negative results }\end{array}$ \\
& $\begin{array}{c}\text { Anxiety and sometimes morbidity } \\
\text { for those with false positive results }\end{array}$ \\
& Hazard of screening test \\
\hline
\end{tabular}


The second benefit is identifying the disease sufficiently early to permit cure by less radical treatment than would be needed if it were allowed to progress to the symptomatic stage. The price paid for this is overtreatment of similar lesions which, if not identified, would not have progressed to overt disease. 'The third benefit is the saving of resources which may benefit the public sector if the need for expensive services used by late cases can be averted, and the private sector by, for example, extending working capacity. Against this must be set the resource costs of the screening procedure itself, the further investigation of positive cases generated by it, and the overtreatment of non-progressive disease.

The reassurance given by a negative screening result is a rather different benefit in that it applies not to the minority found to be diseased but to the great majority of screened subjects. It is a benefit completely independent of whether or not early detection improves prognosis. In at least one documented case the expressed demand from the public for the reassurance given by screening was enough to maintain a screening service whose other benefits were unproven. ${ }^{5}$

The last three disadvantages listed in the table arise from the screening test itself which almost inevitably lacks perfect sensitivity and specificity, thus yielding false negative and false positive results. The false negative patients may ignore symptoms because they feel reassured by their negative test result and may feel resentment and anger when the diagnosis is eventually made. The false positive patients are given needless anxiety and may also suffer physical morbidity from the additional diagnostic investigations (eg, biopsy) needed to establish that they are free from disease. Finally, the tests used for screening may not be entirely safe (eg, amniocentesis).

In ideal theoretical circumstances an evaluation of screening would not only measure the frequency of each of these outcomes of the programme but would also weight each one with an appropriate value, expressed in common units, so that if the sum of units in the benefits column exceeded that in the disadvantages column then screening should be implemented. In practice, of course, it is not possible to achieve this counsel of perfection, due partly to practical difficulties in measuring the frequency of some of the more intangible factors, but mainly to the differing weighting values placed upon them by different members of society.

\section{History of prescriptive screening}

In the field of maternal and child health, a long tradition of screening goes back 60 or 70 years, long before the need for evaluation was appreciated. The raison d'être for antenatal care, for much of infant welfare, and for the school health service is the belief that screening will result in early recognition and treatment of disorders with potentially serious implications. It is difficult now to challenge the value of these long established and revered systems of medical care, but their precise role in bringing down maternal, fetal, infant, and child mortality and morbidity cannot be ascertained, since so many other factors such as nutritional improvements were happening over the same period of time. Attempts to demonstrate the value of these services by comparing outcome in those who use the service and in those who do not is beset by the difficulty so often encountered in screening-a selection into the programme of those least at risk. ${ }^{6}$ Perhaps the most convincing evidence of their value comes from the recent decline in perinatal and infant mortality in France after the introduction of legislation to promote greater uptake of antenatal and neonatal screening, but even in this study other social changes, such as rising standards of education and of housing, were occurring at the same time. ${ }^{7}$

Within the last few decades the enormous development of biochemical, biophysical, and histological diagnostic techniques has made possible early diagnosis of a wide range of disorders in adults as well as in children. This, together with an increasing awareness of the potential disadvantages of screening, has stimulated research into the value of screening for specific disorders. In the remainder of this paper evidence is considered which is currently available on the efficacy of screening for various conditions within the context of morbidity patterns in Britain.

\section{Antenatal screening}

In addition to the traditional role of antenatal care in promoting fetal and maternal wellbeing, various tests for specific fetal abnormalities are now available. These fall into two main categories: (i) those which allow treatment of the fetus in utero or in the immediate neonatal period, and (ii) those which reveal conditions for which there is no treatment and in which prognosis for the fetus is so poor that termination of pregnancy is offered to the prospective parents.

Examples of successful screening programmes of the first type are screening maternal serum for a rising titre of Rhesus antibodies (which has become less necessary now that primary prevention of Rhesus incompatability can be achieved) and screening for syphilis. 
The second type of antenatal screening for fetal defects, where the only "treatment" is euthanasia of the fetus by termination of pregnancy, differs from most other screening programmes in that there is no need to prove the efficacy of treatment. A more important difference arises from moral and ethical considerations, not only about terminating the pregnancy but also about possible damage to normal fetuses. The best way to strike the sometimes difficult balance between potential benefits and potential risks is better decided by the prospective parents than by the doctor or health authority. This means that parents must be fully informed of the consequences of a positive test result, of the chances of having an affected baby, and of harming an unaffected one, implying a need for skilled communication with prospective parents in antenatal clinics.

\section{NEURAL TUBE DEFECTS}

Screening pregnancies for neural tube defects by maternal serum alphafetoprotein (AFP) level has been widely advocated and is practised as a routine in many parts of the country. A raised AFP level in the serum between the 16th and 20th week of gestation is an indication for further investigations firstly by ultrasound, which will verify gestational age, check that the raised level is not due to multiple pregnancy, and visualise anencephaly, and, secondly, by amniocentesis to test amniotic fluid AFP. If this, too, is raised then termination is offered. This screening programme abounds with ethical dilemmas. In the first place, a majority of affected fetuses identified would die anyway, as spontaneous abortions, stillbirths or neonatal deaths, so that termination of these pregnancies is merely advancing by some weeks the date of an inevitable death. Only about 20-25\% would survive as handicapped children, but the physical and, in some cases, mental handicap associated with spina bifida is often very severe and causes immense suffering and distress to the affected children and their families; the main benefit of screening is to avoid the birth of these children. Another difficulty is the lack of specificity of serum AFP levels, which means that 2-3\% of unaffected pregnancies are subjected to amniocentesis, which itself may damage $1-3 \%$ of normal fetuses; ${ }^{8}$ moreover, amniotic fluid AFP may also give false positive results in $0.5 \%$ of normal pregnancies, leading to mistaken terminations. In high incidence areas, such as the west of Scotland, the balance of benefit of averted spina bifida births to risks of damaging unaffected pregnancies is likely to be in favour of routine screening and may also be cost effective. ${ }^{9}$ But, in low risk areas, the balance of benefits to risks is less certain. ${ }^{10}$ One carefully evaluated programme in south Wales-a high incidence area-has shown a reduction of $41 \%$ in the birth prevalence of open spina bifida and a $66 \%$ reduction of anencephaly. ${ }^{11}$ In the country as a whole, there has been an acceleration in the rate at which notified CNS abnormalities have been declining, contemporary with an increase in abortions performed because of suspicion of CNS abnormality, ${ }^{12}$ most of which can be attributed to AFP screening. But the numbers suggest that the contribution of abortions to the total decline is relatively small (figure); this may be because this specific reason is not always notified on the abortion form, or it may be due to a continuing real decline in incidence of the condition, perhaps as a result of improved nutrition of the present generation of parents. Technical improvements in ultrasound examination of the fetus may allow a reduction in the need for amniocentesis and hence a reduction in risk, but ultrasound, too, gives both false negative and false positive results which have not yet been fully evaluated. ${ }^{13}$ Hence the recommendation of the Black committee on this topic, ${ }^{14}$ that screening should be made available for parents who wish to "opt in" to the screening programme, rather than applied routinely, is still relevant.

\section{DOWN'S SYNDROME}

The well known increase in birth prevalence of Down's syndrome with increasing maternal age allows selective screening of older mothers by

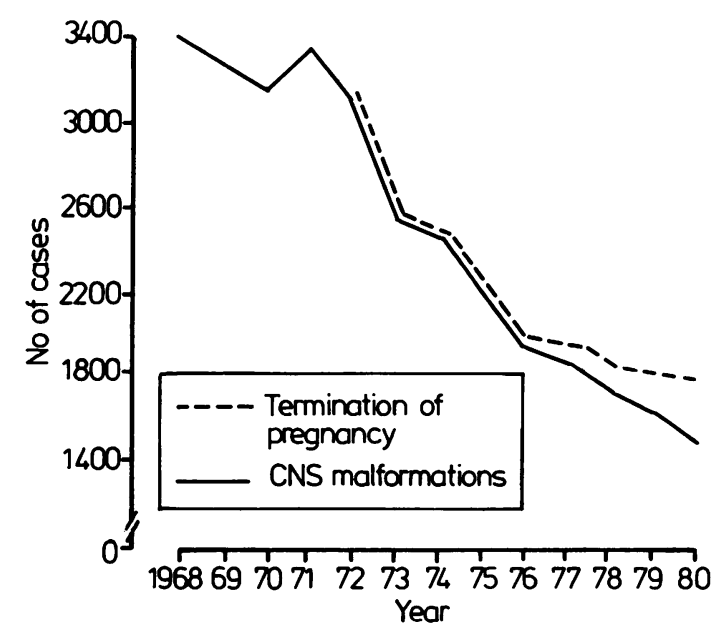

Decline in number of notified births with CNS malformations, showing the projected decline including terminations of pregnancy for suspected CNS malformation. 
amniocentesis and cytogenetic examination of fetal cells. The same considerations about risks of amniocentesis apply as in screening for neural tube defects, but, because screening is offered to only a very small minority of mothers, there is more opportunity to give them a full explanation. The prevalence of Down's syndrome identified at amniocentesis is two to three times greater than the expected birth prevalence, the discrepancy between them presumably being due in part to cases which would have aborted spontaneously if they had not been identified by screening and terminated, and in part to undernotification of Down's syndrome at birth. Thus again much of the effect of the screening programme is to advance an inevitable death. Although many mothers opt for screening as young as 35 , it is only over the age of 40 that the benefits numerically outweigh the risks. The proportion of Down's syndrome births averted by screening depends of course on age specific birth rates; screening every mother aged over 40 would reduce the birth prevalence of Down's syndrome by only $17 \%$, and if the age limit were lowered to $35,35 \%$ of the estimated 1300 births each year could be averted. National figures from birth notifications and terminations of pregnancy for chromosomal abnormalities indicate that the screening programme is having a substantial effect only in mothers over the age of $45,,^{12}$ among whom a two-thirds reduction in birth prevalence has been achieved.

\section{OTHER INHERITED DISORDERS}

Antenatal diagnosis and termination are also offered to selected high risk groups. Screening for $\beta$-thalassaemia by fetal blood sampling may be offered to parents both of whom are heterozygotes for this condition. Parents who have already had one affected child, or in whom there is a strong family history, are identified as a high risk group for screening for chromosomal abnormalities and for sex linked disorders. Considerable effort is being put into antenatal diagnosis of cystic fibrosis, but a test sufficiently specific to warrant fetal blood sampling for this condition is not yet available.

\section{Screening in infancy}

Presymptomatic diagnosis and early treatment of various congenital or acquired disorders in infancy are of generally accepted value, even though a complete evaluation, by comparing the outcome between similar groups who have been offered or not offered screening, has not been done for any of the conditions mentioned below. In most instances, screening for congenital disease need be done only once and hence stands a much better chance of being cost effective than the repeated screens necessary to detect diseases such as cancer acquired in later life.

\section{PHENYLKETONURIA}

Testing blood samples at 6 to 10 days of age for raised levels of phenylalanine has been practised routinely for the past 15 years. Although the condition is rare, the severe mental handicap it entails can be averted by a low phenylalanine diet. The test has a very high sensitivity and specificity, and the organisation of the screening programme successfully reaches $99 \%$ of the population. Only about $1 \%$ of cases are not detected by screening, and the great majority of patients are now of normal intelligence..$^{15}$ Perhaps the only drawback of this screening system is that it "overdiagnoses" and hence overtreats a proportion of patients who, in the absence of screening, would have developed with normal intelligence. This is indicated by an increase in incidence from 0.6 to 0.7 per 10000 before serum screening was introduced to 1 per 10000 now.

\section{CONGENITAL HYPOTHYROIDISM}

Within the last few years, screening neonatal blood for hypothyroidism has been added to the existing system of screening for phenylketonuria, so that one specimen of blood suffices for both tests. A TSH level above $25 \mathrm{~m} \mu / \mathrm{l}$ has a sensitivity of over $95 \%$ and a specificity well over $99 \%$. There is much circumstantial evidence that early treatment with thyroid hormone leads to normal intelligence and normal neurological development. A theoretical cost effectiveness analysis also suggests that there may be a net benefit to the public sector in screening for this condition. ${ }^{16}$ As with phenylketonuria, the yield of cases diagnosed once screening has been introduced (1 in approximately 3000 births) exceeds the previous incidence ( 1 in 6000 births), suggesting an overdiagnosis and overtreatment of some borderline cases.

CONGENITAL DISLOCATION OF THE HIP

In infant care, screening for congenital dislocation of the hip by the Ortolani procedure is widely practised. Using trained staff to screen at birth the test has a sensitivity of $92 \%$ and a specificity of $93 \%$ but it probably suffers from a low repeatability between untrained observers. ${ }^{17}$ The younger the infant, the greater the apparent prevalence, and it is suggested that over half of the unstable hips identified soon after birth will become normal in three weeks. Therefore postponement of screening to around 6 weeks of age is sometimes recommended, but the risk of subsequent dislocation is much greater in those with unstable joints in the neonatal period. 
Confirmation of positive screening findings is by $x$ ray. The efficacy of treatment, whether by plaster immobilisation in an abducted position or by traction and gradual reduction, in preventing the later sequelae of hip disease, has never been tested in a clinical trial but is accepted as being of value. The former treatment method is not without complications, however, leading in some cases to avascular necrosis of the femoral head. The balance of benefits to disadvantages of screening for congenital dislocation of the hip is not proven to be in favour of screening ${ }^{18}$ but, because it has been practised for so long, it may be regarded as unethical to conduct a trial withholding screening from a control group.

\section{SENSORY IMPAIRMENTS}

Screening for deafness in infancy is widely applied and probably leads to earlier correction and hence improved communication, but these benefits are very difficult to quantify. ${ }^{19}$ Similarly, visual screening detects the early signs of squint and, in some cases, facilitates correction by orthoptic exercises rather than surgery and avoids suppression of vision in the affected eye.

\section{OTHER DISORDERS}

General examinations can detect signs of disordered motor development and conditions such as congenital heart disease. The value of presymptomatic detection of these abnormalities is not known. Experimentally, screening may also be carried out for various inherited metabolic diseases, of which cystic fibrosis is the most important. Neonatal screening of blood trypsin levels is capable of diagnosing presymptomatic cystic fibrosis, but until present trials have been completed it is not known if prophylactic antibiotics and physiotherapy are capable of averting the progressive lung damage which eventually proves fatal in this disease. The same heel prick blood sample used for phenylketonuria and hypothyroidism screening can also be used for trypsin levels, so that if early treatment is shown to be of value this could be a highly cost effective programme.

\section{Screening school children}

\section{SENSORY IMPAIRMENTS}

Screening for deafness in school children is normally done by audiometry; deafness in children aged 5 to 15 is more likely to be due to chronic post infective obstruction than to neurological or congenital causes. Although there is some doubt whether treatment of "glue ear" alters its natural history, ${ }^{20}$ it is accepted that children benefit from correction of auditory impairment. Similarly, there is little doubt that visual screening of school children is beneficial in that it permits easy correction by spectacles of most visual impairments.

\section{DENTAL DISEASE}

Detection and treatment of dental caries and correction of orthodontic problems are also accepted as being of value, particularly in view of the high prevalence of these conditions. It is possible that dental screening also contributes to primary prevention, for example by fluoride application, recommendation of fluoride toothpaste, and, less certainly, dietary advice.

\section{SKIN DISEASES}

Screening for skin diseases such as head lice is of more obvious value in containing epidemics and thus protecting unaffected children than as a form of prescriptive screening. The same principle underlies the widespread practice of screening for plantar warts, even though their transmission in the precincts of swimming pools is unproven. One cynic has even suggested that this screening programme increases mortality from drowning among children who, because of plantar warts, have been deprived of swimming lessons. ${ }^{21}$

SCOLIOSIS

There is currently considerable demand from 8 orthopaedic surgeons for screening adolescents for scoliosis. The screening procedure advocated iso visual inspection (with a relatively low specificity of around $90 \%$ ) followed by $x$ ray. About $2 \%$ of children are found to have some degree of scoliosis, but in only $0 \cdot 2 \%$ is treatment by a spinal brace or fusion recommended, the remainder being treated by exercises. There is considerable doubt about the efficacy of treatment, and probably a majority of cases are, in any case, non-progressive. This is clearly a form of screening that requires an evaluative trial, ${ }^{22}$ preferably by randomly allocating children (or schools) to a screening group or a control group, and following up all subjects for a sufficient number of years to observe differences in morbidity from scoliosis. Regrettably, some authorities are already including scoliosis screening in their routine school health service even though this evaluation of its benefits and disadvantages has not been done.

\section{Screening adults}

Containment by screening of morbidity and mortality from major neoplastic and vascular diseases prevalent in middle age in developed countries has so far proved disappointing. The insidious onset and 
usually long natural history of these conditions make it difficult to diagnose precisely which individuals are affected by progressive disease, and even more difficult to show that early treatment gives better long term results than treatment of symptomatic disease. Unlike infants and school children, for whom systems exist to reach the great majority of the target population, adult screening is dependent on people's knowledge of, and willingness to participate in, the screening programme; poor compliance by the target population may severely limit the effectiveness of a screening programme.

\section{CANCER OF THE CERVIX}

Although screening by cervical cytology has been practised for 20 years, it is only very recently that convincing evidence of its potential benefit has been published. This comes from a comparison of the trend in incidence in various Nordic countries which have had different intensities of screening; ${ }^{23}$ a $50-70 \%$ fall in incidence has occurred in countries such as Iceland and Finland in which $70 \%$ or more of the eligible population have been screened, but not in Norway where population screening has been very limited. There has been no fall in incidence in Britain, where the screening system has failed to reach two-thirds of the women who develop invasive cancer. Thus a screening service that is of potential value is not achieving its objective, largely because women past their childbearing years, who are at increased risk, are not being screened. ${ }^{24}$ The principal disadvantage of screening for cervical cancer is probably overdiagnosis of non-progressive cervical intraepithelial neoplasia, particularly in young women. The natural history of progression from intraepithelial to invasive neoplasia is so uncertain that the progressive cases cannot be distinguished from the rest, so all must be treated.

\section{CANCER OF THE BREAST}

A justly renowned randomised controlled trial of screening for breast cancer has shown a one third reduction in mortality in a group of women aged 45-64 who were offered screening by clinical examination, compared with a control group. ${ }^{25} \mathrm{~A}$ number of questions remain unanswered, however, including the value of screening women under 50 , the independent contributions of clinical examination and mammography, and the effectiveness of breast self-examination as a screening test. These are currently the subject of a number of European and Canadian trials which should begin to show mortality differences, if any, within the next few years. Meanwhile, two uncontrolled population based studies in the Netherlands have demonstrated a $50-70 \%$ reduction in risk of dying from breast cancer among screened women compared with unscreened women from the same population. ${ }^{28}$ The disadvantages of screening for breast cancer include the identification of borderline intraepithelial neoplasia (although this is not as large a problem as in cervical cancer screening); false positive results lead to possible anxiety and an increased biopsy rate; and costs that are likely to be heavy because of the need to repeat screening at relatively short intervals, for example, annually. Concern about the potential radiation hazard of mammography has led to a reduction of the radiation dose to well under $0.5 \mathrm{rad}$ per examination, with a negligible risk. It is still desirable, however, to monitor the dose from mammography systems at regular intervals.

\section{OTHER CANCERS}

Population screening for other cancer sites is still experimental. ${ }^{27}$ The potential for benefit from faecal occult blood testing for large bowel tumours appears good, subject to increasing compliance from people aged over 45; it will, however, be some years before the results of randomised controlled trials of screening show whether mortality can be lowered. Various controlled trials of screening for lung cancer by sputum cytology and chest $x$ ray have so far shown no benefit. Screening for stomach cancer by barium meal $x$ ray is widely practised in Japan but has not been evaluated. Screening for bladder cancer by urinary cytology and haematology is also largely unevaluated, although it is offered as a service to men who have worked in the rubber and dye industries where there is a special risk.

\section{CEREBROVASCULAR DISEASE}

Screening blood pressure levels to detect and treat people at high risk of subsequent stroke is of potential value. Early clinical trials of treatment of men with diastolic levels above $115 \mathrm{~mm} \mathrm{Hg}$ showed a substantial decrease in incidence of and mortality from stroke, ${ }^{28}$ which was subsequently confirmed by workers in Australia. ${ }^{29}$ However, since only a minority of those with raised blood pressure will suffer a stroke, the wisdom of long term drug treatment of large numbers of people has been questioned. ${ }^{30}$ Various non-pharmacological methods, such as weight reduction and salt restriction, are advocated but their efficacy in reducing the incidence of disease is unproven. One American study has shown a reduction in overall mortality in people with blood pressure levels above $90 \mathrm{~mm} \mathrm{Hg}$, randomly allocated to "stepped-up" care or to a control group, but the reduction was greatest in those with the lowest blood pressure levels and applied more to non-cardiovascular mortality, suggesting that the difference was not specifically 
caused by better control of hypertension. ${ }^{31}$ Until the results of the current Medical Research Council hypertension trial, which is looking at the efficacy and side effects of different drugs within specified blood pressure levels and age/sex groups, are available, it cannot be claimed with confidence that the benefits of screening for stroke outweigh the disadvantages.

\section{CORONARY HEART DISEASE}

Multiple risk factor intervention trials which have aimed to reduce myocardial infarctions by lowering blood pressure, lowering saturated fat intake, and reducing smoking have been inconclusive. But there is increasing evidence that reduction of asymptomatic hypercholesterolaemia, particularly low density lipoprotein (LDL) cholesterolaemia, does result in a lower incidence of and mortality from coronary heart disease. ${ }^{32}$ Trials of dietary advice and of drug treatment by clofibrate or cholestyramine have all shown reductions in risk in men with very high cholesterol levels in the top $5-20 \%$ of the distribution. But the cholesterol-lowering drugs-particularly clofibrate- require further long term study to evaluate their potentially harmful side effects.

Screening cholesterol levels to identify those in the top quintile and to offer them a dietary regime sufficient to reduce their LDL cholesterol by $15 \%$ over many years can now be justified. So far, however, the trials have been on highly selected subjects, and the feasibility of extending this form of screening to the general population requires further operational study. In particular, information on resource requirements for implementing dietary regimes, and for the follow-up of subjects, is needed. It is to be hoped that primary prevention by changing national dietary and smoking habits may in the long term make screening for raised cholesterol levels unnecessary, except perhaps in very high risk groups such as those with familial hypercholesterolaemia.

\section{OTHER CONDITIONS}

Screening middle aged adults for numerous other diseases, such as diabetes, anaemia, urinary infections, glaucoma, and various biochemical abnormalities, has been tried experimentally but not found to be of sufficient value to be applied on a routine basis to unselected populations. Screening questionnaires for anxiety and depression have been validated, and trials of psychotropic drug treatment in those patients detected have been shown to be useful in selected high risk groups, for example, postmastectomy patients, but their value in the general population is largely untried.

\section{Screening in old age}

The prevalence of multiple unreported diseases in elderly people is well recognised. It is doubtful, however, whether detection and treatment of diseases such as those mentioned in the section above are of any real value in the elderly, since the aim of health services in this age group is more to improve the quality of life than to extend its duration. Screening to identify conditions affecting the individual's ability to carry on living independently is therefore more relevant, and there are many instances of multiple screening for disorders of locomotion, vision, hearing, nutrition, and so on, often applied by health visitors on a domiciliary basis. ${ }^{33}$ Measures of the outcome of treatments to alleviate these conditions are less clear cut than incidence and mortality data which are available for evaluation of screening in younger age groups. This, together with non-compliance with the treatments offered, accounts for the fact that, despite its intuitive "caring" appeal, factual evidence of the benefit of screening the elderly is still lacking.

\section{Conclusion}

Most of the disorders for which screening can clearly be recommended are in children. The benefits are more obvious in early life, compliance (of parents) is better, and the costs are considerably less, particularly for congenital disorders where a single examination can establish whether or not the subjecto is affected. This is in contrast to diseases of adult life? where, in view of the usual pattern of increasing incidence with increasing age, repeated screening at intervals is needed. Screening infants for phenylketonuria, hypothyroidism, visual and auditory impairments and, probably, congenital dislocation of the hip is of value, and-subject to the parents' decision to participate-the same is true of antenatal screening for Down's syndrome and neural tube defects in high incidence populations. Screening school children for visual, auditory, and dental disease is worth while. Children's screening programmes that have not yet been proved to be of value include those for cystic fibrosis and scoliosis.

Evaluation of screening for chronic diseases in adults has proved more difficult and lengthy than was first supposed, and it can still be clearly recommended only for cervical cancer. Evidence is, however, accumulating that screening for breast cancer, severe hypercholesterolaemia, and possibly hypertension is probably worth while.

Two problems are encountered in implementing almost every population screening programme. The first is to convince the target population that 
screening is relevant and valuable to them, hence increasing compliance both with screening and with subsequent treatment. The second is to decide the management of borderline abnormalities identified by screening. The extent of "overtreatment" can be determined only by a long term follow-up comparing a screened or treated group with a control group. Although sometimes termed secondary prevention, screening, as a strategy for controlling disease, comes a rather poor third after primary prevention-the winner-and effective treatment of established disease-in second place. This is because of the considerable effort, cost, and possible morbidity it entails for society at large and for screened individuals in particular, only a tiny minority of whom will achieve through it an improved prognosis.

\section{References}

${ }^{1}$ McKeown T. Validation of screening procedures. In: Screening in medical care, published for the Nuffield Provincial Hospitals Trust. London: Oxford University Press, 1968: 1-13.

${ }^{2}$ Simpson PR. Economic aspects of screening. In: Report of the first UICC workshop on evaluation of screening programmes for cancer. UICC Technical Report. Geneva: in press.

${ }^{3}$ Wilson JMG, Jungner G. The principles and practice of screening for disease. Public Health Papers 34. Geneva: WHO, 1968.

${ }^{4}$ Cochrane AL, Holland WW. Validation of screening procedures. Br Med Bull 1971; 27: 3-8.

${ }^{5}$ Hansard. Parliamentary debates. House of Commons Official Report, 1982; 33: Col. 78.

${ }^{6}$ Bloor MJ, Gill DG. Screening of the well child. Community Medicine 1972; 129: 135-8.

${ }^{7}$ Rumeau-Rouquette C, Crost-Deniel M, Breart G, du Mazaubrun C. Evaluation epidemiologique du programme de santé en périnatologie. I Region Rhône-Alpes 1972-1975. Rev Epidemiol Med Soc Santé Publique 1977; 25: 107-29.

${ }^{8}$ Medical Research Council Working Party on Amniocentesis. An assessment of the hazards of amniocentesis. Br J Obstet Gynaec 1978; 85: suppl 2, $1-41$.

${ }^{9}$ Hagard S, Carter FA, Milne RC. Screening for spina bifida cystica: a cost benefit analysis. Br J Prev Soc Med 1978; 30: 40 .

${ }^{10}$ Chamberlain J. Human costs and benefits of screening for neural tube defects. Lancet 1978; ii: 1293-6.

${ }^{11}$ Roberts CJ, Hibbard BM, Elder GH, et al. The efficacy of a serum screening service for neural tube defects: the South Wales experience. Lancet 1983; i: 1315-8.

12 Weatherall JAC. A review of some effects of recent medical practices in reducing the numbers of children born with congenital abnormalities. Health Trends 1982; 14: 85-8.
${ }^{13}$ Roberts CJ, Evans KT, Hibbard BM, Laurence KM, Roberts EE, Robertson IB. Diagnostic effectiveness of ultrasound in detection of neural tube defect. Lancet 1983; ii: 1068-9.

14 Working Group on Screening for Neural Tube Defects. Department of Health and Social Security (Black Report) 1979.

${ }^{15}$ Medical Research Council Steering Committee for the MRC/DHSS Phenylketonuria Register. Routine neonatal screening for phenylketonuria in the United Kingdom, 1964-78. Br Med J 1981; 282: 1680-4.

${ }^{16}$ Smith P, Morris A. Assessment of a programme to screen the newborn for congenital hypothyroidism. Community Medicine 1979; 1: 14-22.

${ }^{17}$ Cunningham KT, Beningfield SA, Moulton A, Maddock CR. A clicking hip in a newborn baby should never be ignored. Lancet 1984; i: 668-70.

${ }^{18}$ Parkin DM. How successful is screening for congenital disease of the hip? Am J Public Health 1981; 71: 1378-83.

${ }^{19}$ Knox EG. Deafness in childhood. In: Screening in medical care, published for the Nuffield Provincial Hospitals Trust. London: Oxford University Press, 1968: 55-64.

${ }^{20}$ Black N. Epidemiology of glue ear. Submission for Part II Membership of the Faculty of Community Medicine.

${ }^{21}$ Tudor Hart J. Paper presented to the Royal Society of Medicine, 31 January 1984

${ }^{22}$ Leaver JM, Alvik A, Warren MD. Prescriptive screening for adolescent idiopathic scoliosis; a review of the evidence. Int J Epidemiol 1982; 11: 101-11.

${ }^{23}$ Hakama M. Trends in the incidence of cervical cancer in the Nordic countries. In: Magnus K, ed. Trends in cancer incidence, causes and practical implications. Washington: Hemisphere Publishing Corporation, 1982: 279-92.

${ }^{24}$ Draper G. Screening for cervical cancer: revised policy. Health Trends 1982; 14: 37-40.

${ }^{25}$ Shapiro S, Venet W, Strax P, Venet L, Roeser R. Ten to fourteen year effects of breast cancer screening on mortality. J Nat Cancer Inst 1982; 69: 349-55.

${ }^{26}$ Editorial. New evidence on the value of breast screening. Lancet 2 June 1984.

${ }^{27}$ Chamberlain J. Screening for cancer. Community Medicine 1983; 5: 283-6.

${ }^{28}$ United States Veterans' Administration Co-operative Study Group on Anti-hypertensive Agents. Effects of treatment on morbidity in hypertension. JAMA 1970; 213: 1143.

${ }^{29}$ The Australian Therapeutic Trial in Mild Hypertension. Report by the Management Committee. Lancet 1980; i: 1261-7.

${ }^{30}$ Oliver MF. Risks of correcting the risks of coronary disease and stroke with drugs. New Engl J Med 1982; 306: 297-8.

${ }^{31}$ The Lipid Research Clinics Coronary Primary Prevention Trial Results. I Reduction in incidence of coronary heart disease. II Relationship of reduction in incidence of coronary heart disease to cholesteral lowering. JAMA 1984; 251: 351-74.

${ }^{32}$ Oliver MF. Hypercholesterolaemia and coronary heart disease: an answer. $\mathrm{Br} \mathrm{Med} J$ 1984; 288: 423-4.

${ }^{33}$ Lowther CP, Macleod RDM, Williamson J. Evaluation of early diagnostic services for the elderly. Br Med J 1970; 3: 275-7. 